\title{
COMPUTING ZAGREB INDICES AND ZAGREB POLYNOMIALS OF FULLERENE, BUTTERFLY AND BENES NETWORKS
}

\author{
Jianzhong $\mathrm{Xu}^{1}$, Muhammad K. Siddiqui ${ }^{2}$, \\ Mohammad R. Farahani ${ }^{3}$, Ismail Naci Cangul ${ }^{4} \S$ \\ ${ }^{1}$ Department of Electronics and Information Engineering \\ Bozhou University, Bozhou 236800, CHINA \\ ${ }^{2}$ Department of Mathematics \\ COMSATS University Islamabad \\ Lahore Campus, 54000, PAKISTAN \\ 3 Department of Applied Mathematics \\ Iran University of Science and Technology (IUST) \\ Narmak, Tehran 16844, IRAN \\ ${ }^{4}$ Bursa Uludag University, Mathematics \\ 16059, Bursa, TURKEY
}

\begin{abstract}
A topological index is a numerical parameter of a graph which characterizes some of the topological properties of the graph. The concepts of hyper-Zagreb index, first multiple Zagreb index, second multiple Zagreb index and relatedly the Zagreb polynomials were established in chemical graph theory by means of the vertex degrees. It is reported that these indices are useful in the study of anti-inflammatory activities of certain chemical networks. In this paper, we study fullerene, butterfly, Benes networks and determine the hyperZagreb index, first multiple Zagreb index, second multiple Zagreb index and the Zagreb polynomials of them.
\end{abstract}

AMS Subject Classification: 05C12, 37B30

Key Words: hyper-Zagreb index, multiple Zagreb index, Zagreb polynomial, fullerene, butterfly, Benes network

Received: June 17, 2019

(c) 2019 Academic Publications

$\S$ Correspondence author 


\section{Introduction}

Graph theory is a branch of mathematics which provides useful tools such as topological indices for chemists. Molecules and molecular compounds are often modeled by a molecular graph. A molecular graph is a representation of the structural formula of a chemical compound in terms of graph theory, whose vertices correspond to the atoms of the compound and edges correspond to chemical bonds. A graph $G(V, E)$ with vertex set $V$ and edge set $E$ is connected if there exists a path between any pair of vertices in $G$. A network is simply a connected graph having no multiple edges nor loops. For a graph $G$, the degree of a vertex $w$ is the number of edges incident to $w$ and denoted by $\operatorname{deg}(w)$.

A graph can sometimes be recognized by a numeric number, a polynomial, a sequence of numbers or a matrix which represents the whole graphs in several ways, and these representations are aimed to be uniquely defined for that graph which is not possible always. A topological index is a number associated with a graph which verifies the topology of the graph and is invariant under graph automorphisms. Recently, some types of topological indices such as distance-based topological indices, degree-based topological indices, etc. have been defined and related polynomials and indices of graphs have been intensively studied. Among these types of indices, degree based topological indices have great importance and play a vital role in chemical graph theory as the number of chemical bonds at each atom corresponds to the degree of the vertex in the modeling graph. In a more precise way, a topological index $\operatorname{Top}(G)$ of a graph $G$ is a number with the property that for every graph $H$ isomorphic to $G$, $\operatorname{Top}(H)=\operatorname{Top}(G)$. The first known use of the concept of topological index was the work done by Wiener, [40], while he was working with the boiling points of paraffins. He named this index as path number. Later on, the path number was renamed as Wiener index due to its importance and applications. The Wiener index is the first and most studied topological index, both from theoretical point of view and applications, and defined as the sum of distances between all pairs of vertices in $G$, see for details [9, 21].

Two of the oldest topological indices are the first and second Zagreb indices introduced by I. Gutman and N. Trinajstic in 1972, are also based on degrees of vertices of $G,[22]$. The first and second Zagreb indices of a graph $G$ are defined by

$$
M_{1}(G)=\sum_{w_{1} w_{2} \in E(G)}\left[\operatorname{deg}\left(w_{1}\right)+\operatorname{deg}\left(w_{2}\right)\right]
$$


and

$$
M_{2}(G)=\sum_{w_{1} w_{2} \in E(G)}\left[\operatorname{deg}\left(w_{1}\right) \times \operatorname{deg}\left(w_{2}\right)\right] .
$$

The reader can find further information about these two indices in $[2,5,7,20$, 23, 28, 35, 38, 39]. In 2013, G. H. Shirdel, H. Rezapour and A. M. Sayadi, [36], introduced a new degree based Zagreb index named "hyper-Zagreb index" by

$$
H M(G)=\sum_{w_{1} w_{2} \in E(G)}\left[\operatorname{deg}\left(w_{1}\right)+\operatorname{deg}\left(w_{2}\right)\right]^{2} .
$$

M. Ghorbani and N. Azimi defined two new versions of Zagreb indices of a graph $G$ in 2012, [16]. The first multiple Zagreb index $P M_{1}(G)$ and second multiple Zagreb index $P M_{2}(G)$ are defined by

$$
P M_{1}(G)=\prod_{w_{1} w_{2} \in E(G)}\left[\operatorname{deg}\left(w_{1}\right)+\operatorname{deg}\left(w_{2}\right)\right]
$$

and

$$
P M_{2}(G)=\prod_{w_{1} w_{2} \in E(G)}\left[\operatorname{deg}\left(w_{1}\right) \times \operatorname{deg}\left(w_{2}\right)\right] .
$$

The properties of $P M_{1}(G)$ and $P M_{2}(G)$ indices for some chemical structures have been studied in $[10,11,12,14,16]$.

The first Zagreb polynomial $M_{1}(G, x)$ and the second Zagreb polynomial $M_{2}(G, x)$ are defined by

$$
M_{1}(G, x)=\sum_{w_{1} w_{2} \in E(G)} x^{\left[\operatorname{deg}\left(w_{1}\right)+\operatorname{deg}\left(w_{2}\right)\right]}
$$

and

$$
M_{2}(G, x)=\sum_{w_{1} w_{2} \in E(G)} x^{\left[\operatorname{deg}\left(w_{1}\right) \times \operatorname{deg}\left(w_{2}\right)\right]} .
$$

The properties of $M_{1}(G, x)$ and $M_{2}(G, x)$ polynomials for some chemical structures have been studied in [19].

Nowadays there is an extensive research activity on $H M(G), P M_{1}(G)$, $P M_{2}(G)$ indices and $M_{1}(G, x)$ and $M_{2}(G, x)$ polynomials and their variants, see also $[12,15,16,18,22,25,36]$.

For further study on topological indices of various graph families, see $[1,13$, $17,25,24,26,29,33,34,37]$. 


\section{Butterfly networks}

Butterfly graphs are defined as the underlying graphs of Fast Fourier Transforms (FFT) networks which can perform the FFT very efficiently. The butterfly network consists of a series of switch stages and interconnection patterns, which allows ' $n$ ' inputs to be connected to ' $n$ ' outputs. The Benes network consists of back-to-back butterflies, [41]. As butterfly is known for FFT, Benes is known for permutation routing, [4]. The butterfly and Benes networks are important multi-stage interconnection networks which possess attractive topologies for communication networks, [33]. They have been used in parallel computing systems such as IBM, SP1/SP2, MIT Transit Project, NEC Cenju-3 and used as well in the internal structures of optical couplers, [32, 42]. The multistage networks have long been used as communication networks for parallel computing, [31].

\subsection{Results for butterfly networks}

One of the most popular bounded-degree derivative networks of the hypercube is the butterfly network. The set $V$ of vertices of an $r$-dimensional butterfly network corresponds to pairs $[w, i]$, where $i$ is the dimension or level of a node $(0 \leq i \leq r)$ and $w$ is an $r$-bit binary number that denotes the row of the node. Two nodes $[w, i]$ and $\left[w^{\prime}, i^{\prime}\right]$ are linked by an edge if and only if $i^{\prime}=i+1$ and either

- $w$ and $w^{\prime}$ are identical, or

- $w$ and $w^{\prime}$ differ in precisely the $i$-th bit.

The edges in the network are undirected. An $r$-dimensional butterfly network is denoted by $B F(r)$. Manuel et al., [33], proposed the diamond representations of these networks. The normal and diamond representations of 3-dimensional butterfly network are given in Fig. 1. The vertex and edge cardinalities are $2^{r}(r+1)$ and $r 2^{r+1}$, respectively.

We compute hyper-Zagreb index $H M(G)$, first multiple Zagreb index $P M_{1}(G)$, second multiple Zagreb index $P M_{2}(G)$ and Zagreb polynomials $M_{1}(G, x)$ and $M_{2}(G, x)$ for butterfly network $B F(r)$.

Theorem 1. Let $B F(r)$ be a butterfly network. Then

$$
H M(B F(r))=36\left(2^{r+2}\right)+64\left(2^{r+1}(r-2)\right)
$$




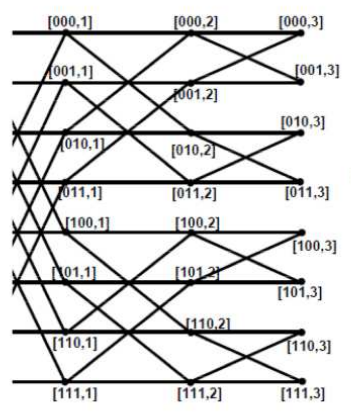

(a):

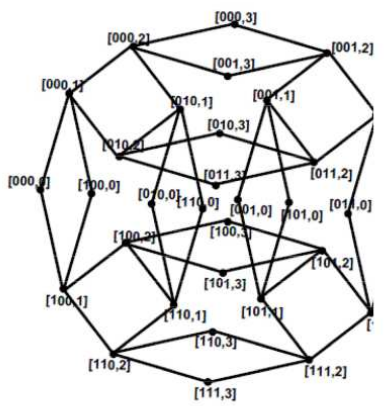

(b):

Figure 1: (a) Normal representation of butterfly $B F(3)$, (b) Diamond representation of butterfly $B F(3)$

$$
\begin{aligned}
P M_{1}(B F(r)) & =6^{\left(2^{r+2}\right)} 8^{\left(2^{r+1}(r-2)\right)} \\
P M_{2}(B F(r)) & =8^{\left(2^{r+2}\right)} 16^{\left(2^{r+1}(r-2)\right)} \\
M_{1}(B F(r), x) & =2^{r+2} x^{6}+2^{r+1}(r-2) x^{8} \\
M_{2}(B F(r), x) & =2^{r+2} x^{8}+2^{r+1}(r-2) x^{16} .
\end{aligned}
$$

Proof. Let $G=B F(r)$ be a butterfly network of dimension $r$. The number of vertices and edges in $B F(r)$ are $2^{r}(r+1)$ and $r 2^{r+1}$, respectively. The edge set $E(B F(r))$ can be partitioned into two sets based on degrees of the end vertices. The first edge partition $E_{1}(B F(r))$ contains $2^{r+2}$ edges $w_{1} w_{2}$ where $\operatorname{deg}\left(w_{1}\right)=2, \operatorname{deg}\left(w_{2}\right)=4$ and the second edge partition $E_{2}(B F(r))$ contains $2^{r+1}(r-2)$ edges $w_{1} w_{2}$ where $\operatorname{deg}\left(w_{1}\right)=\operatorname{deg}\left(w_{2}\right)=4$. Now using equations (1)-(5), we have

$$
H M(B F(r))=\sum_{w_{1} w_{2} \in E(B F(r))}\left[\operatorname{deg}\left(w_{1}\right)+\operatorname{deg}\left(w_{2}\right)\right]^{2}
$$




$$
\begin{aligned}
& =\sum_{w_{1} w_{2} \in E_{1}(B F(r))}\left[\operatorname{deg}\left(w_{1}\right)+\operatorname{deg}\left(w_{2}\right)\right]^{2} \\
& +\sum_{w_{1} w_{2} \in E_{2}(B F(r))}\left[\operatorname{deg}\left(w_{1}\right)+\operatorname{deg}\left(w_{2}\right)\right]^{2} \\
& =6^{2}\left|E_{1}(B F(r))\right|+8^{2}\left|E_{2}(B F(r))\right| \\
& =36\left(2^{r+2}\right)+64\left(2^{r+1}(r-2)\right) \text {, } \\
& P M_{1}(B F(r))=\prod_{w_{1} w_{2} \in E(B F(r))}\left[\operatorname{deg}\left(w_{1}\right)+\operatorname{deg}\left(w_{2}\right)\right] \\
& =\prod_{w_{1} w_{2} \in E_{1}(B F(r))}\left[\operatorname{deg}\left(w_{1}\right)+\operatorname{deg}\left(w_{2}\right)\right] \\
& \times \quad \prod_{w_{1} w_{2} \in E_{2}(B F(r))}\left[\operatorname{deg}\left(w_{1}\right)+\operatorname{deg}\left(w_{2}\right)\right] \\
& =6^{\left|E_{1}(B F(r))\right|} 8^{\left|E_{2}(B F(r))\right|} \\
& =6^{\left(2^{r+2}\right)} 8^{\left(2^{r+1}(r-2)\right)} \text {, } \\
& P M_{2}(B F(r))=\prod_{w_{1} w_{2} \in E(B F(r))}\left[\operatorname{deg}\left(w_{1}\right) \times \operatorname{deg}\left(w_{2}\right)\right] \\
& =\prod_{w_{1} w_{2} \in E_{1}(B F(r))}\left[\operatorname{deg}\left(w_{1}\right) \times \operatorname{deg}\left(w_{2}\right)\right] \\
& \times \quad \prod\left[\operatorname{deg}\left(w_{1}\right) \times \operatorname{deg}\left(w_{2}\right)\right] \\
& w_{1} w_{2} \in E_{2}(B F(r)) \\
& =8^{\left|E_{1}(B F(r))\right|} 16^{\left|E_{2}(B F(r))\right|} \\
& =8^{\left(2^{r+2}\right)} 16^{\left(2^{r+1}(r-2)\right)} \text {, }
\end{aligned}
$$

and hence the corresponding polynomials are

$$
\begin{aligned}
M_{1}(B F(r), x)= & \sum_{w_{1} w_{2} \in E(B F(r))} x^{\left[\operatorname{deg}\left(w_{1}\right)+\operatorname{deg}\left(w_{2}\right)\right]}, \\
M_{1}(B F(r), x)= & \sum_{w_{1} w_{2} \in E_{1}(B F(r))} x^{\left[\operatorname{deg}\left(w_{1}\right)+\operatorname{deg}\left(w_{2}\right)\right]} \\
& +\sum_{w_{1} w_{2} \in E_{2}(B F(r))} x^{\left[\operatorname{deg}\left(w_{1}\right)+\operatorname{deg}\left(w_{2}\right)\right]}
\end{aligned}
$$




$$
\begin{aligned}
& =\sum_{w_{1} w_{2} \in E_{1}(B F(r))} x^{6}+\sum_{w_{1} w_{2} \in E_{2}(B F(r))} x^{8} \\
& =\left|E_{1}(B F(r))\right| x^{6}+\left|E_{2}(B F(r))\right| x^{8} \\
& =2^{r+2} x^{6}+2^{r+1}(r-2) x^{8}, \\
M_{2}(B F(r), x) & =\sum_{w_{1} w_{2} \in E(B F(r))} x^{\left[\operatorname{deg}\left(w_{1}\right) \times \operatorname{deg}\left(w_{2}\right)\right]} \\
& =\sum_{w_{1} w_{2} \in E_{1}(B F(r))} x^{\left[\operatorname{deg}\left(w_{1}\right) \times \operatorname{deg}\left(w_{2}\right)\right]} \\
& \sum_{w_{1} w_{1} w_{2} \in E_{2}(B F(r))} x^{\left[\operatorname{deg}\left(w_{1}\right) \times \operatorname{deg}\left(w_{2}\right)\right]} \\
& \sum_{w_{1} \in E_{1}(B F(r))} x_{E_{1}(B F(r))\left|x^{8}+\right| E_{2}(B F(r)) \mid x^{16}} x^{16} \\
= & 2^{r+2} x^{8}+2^{r+1}(r-2) x^{16} .
\end{aligned}
$$

\subsection{Results for Benes networks}

An $r$-dimensional Benes network is nothing but back-to-back butterflies. An $r$ dimensional Benes network has $2 r+1$ levels, each level with $2 r$ nodes. The level 0 to level $r$ nodes in the network form an $r$-dimensional butterfly. The middle level of the Benes network is shared by these butterflies. An $r$-dimensional Benes network is denoted by $B(r)$. Manuel et al. alternatively proposed the diamond representation of the Benes network, [33]. Fig. 2 shows the normal representation of $B(3)$ network, while diamond representation of $B(3)$ is depicted in Fig. 3. The number of vertices and number of edges in an $r$-dimensional Benes network are $2^{r}(2 r+1)$ and $r 2^{r+2}$, respectively, [29].

We compute the hyper-Zagreb index $H M(G)$, first multiple Zagreb index $P M_{1}(G)$, second multiple Zagreb index $P M_{2}(G)$ and also the Zagreb polynomials $M_{1}(G, x)$ and $M_{2}(G, x)$ for a Benes network of dimension $r$.

Theorem 2. Let $B(r)$ be a Benes network of dimension $r$. Then

$$
\begin{aligned}
& H M(B(r))=36\left(2^{r+2}\right)+64\left(2^{r+2}(r-1)\right) \\
& P M_{1}(B(r))=6^{\left(2^{r+2}\right)} \times 8^{\left(2^{r+2}(r-1)\right)}
\end{aligned}
$$



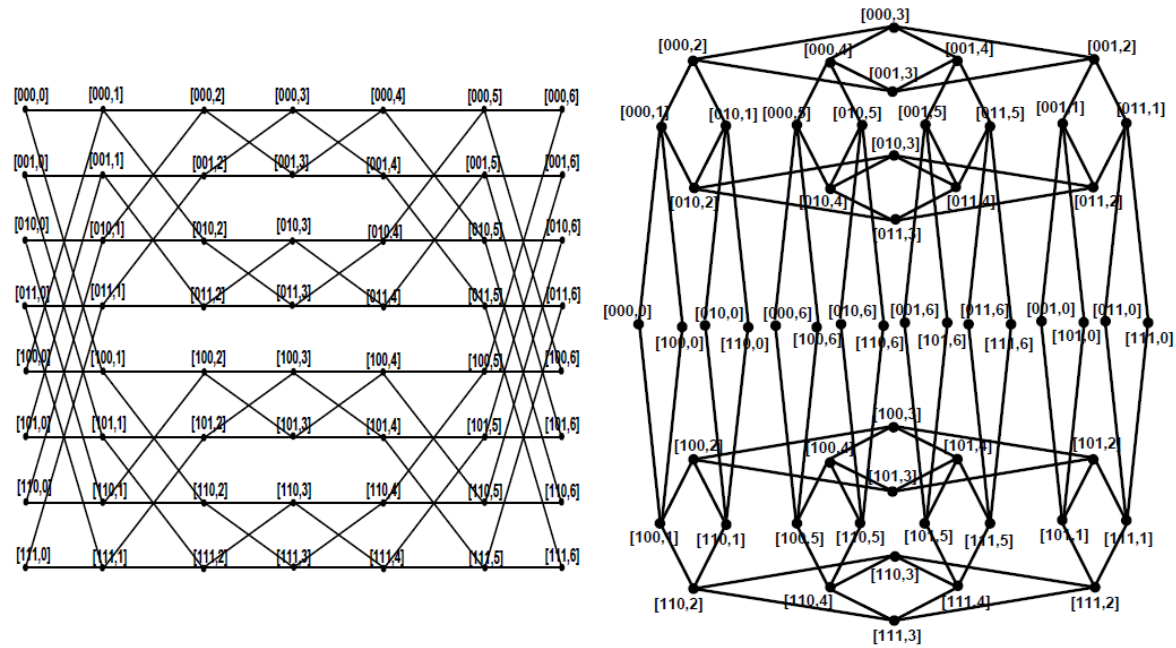

Figure 2: Normal and Diamond representations of Benes network $B(3)$

$$
\begin{aligned}
P M_{2}(B(r)) & =8^{\left(2^{r+2}\right)} \times 16^{\left(2^{r+2}(r-1)\right)} \\
M_{1}(B(r), x) & =2^{r+2} x^{6}+2^{r+2}(r-1) x^{8} \\
M_{2}(B(r), x) & =2^{r+2} x^{8}+2^{r+2}(r-1) x^{16}
\end{aligned}
$$

Proof. Let $G=B(r)$ be a Benes network of dimension $r$. The number of vertices and edges in $B(r)$ are $2^{r}(r+1)$ and $r 2^{r+2}$, respectively. The edge set $E(B(r))$ can be partitioned into two sets based on degrees of the end vertices. The first edge partition $E_{1}(B(r))$ contains $2^{r+2}$ edges $w_{1} w_{2}$ where $\operatorname{deg}\left(w_{1}\right)=$ $2, \operatorname{deg}\left(w_{2}\right)=4$. The second edge partition $E_{2}(B(r))$ contains $2^{r+2}(r-1)$ edges $w_{1} w_{2}$ where $\operatorname{deg}\left(w_{1}\right)=\operatorname{deg}\left(w_{2}\right)=4$. Using equations (1)-(5), we have

$$
\begin{aligned}
H M(B(r)) & =\sum_{w_{1} w_{2} \in E(G)}\left[\operatorname{deg}\left(w_{1}\right)+\operatorname{deg}\left(w_{2}\right)\right]^{2} \\
& =\sum_{w_{1} w_{2} \in E_{1}(B(r))}\left[\operatorname{deg}\left(w_{1}\right)+\operatorname{deg}\left(w_{2}\right)\right]^{2} \\
& +\sum_{w_{1} w_{2} \in E_{2}(B(r))}\left[\operatorname{deg}\left(w_{1}\right)+\operatorname{deg}\left(w_{2}\right)\right]^{2} \\
& =6^{2}\left|E_{1}(B(r))\right|+8^{2}\left|E_{2}(B(r))\right| \\
& =36\left(2^{r+2}\right)+64\left(2^{r+2}(r-1)\right),
\end{aligned}
$$




$$
\begin{aligned}
& P M_{1}(B(r))=\prod_{w_{1} w_{2} \in E(B(r))}\left[\operatorname{deg}\left(w_{1}\right)+\operatorname{deg}\left(w_{2}\right)\right] \\
& =\prod_{w_{1} w_{2} \in E_{1}(B(r))}\left[\operatorname{deg}\left(w_{1}\right)+\operatorname{deg}\left(w_{2}\right)\right] \\
& \times \quad \prod_{w_{1} w_{2} \in E_{2}(B(r))}\left[\operatorname{deg}\left(w_{1}\right)+\operatorname{deg}\left(w_{2}\right)\right] \\
& =6^{\left|E_{1}(B(r))\right|} 8^{\left|E_{2}(B(r))\right|}=6^{\left(2^{r+2}\right)} 8^{\left(2^{r+2}(r-1)\right)}, \\
& P M_{2}(B(r))=\prod_{w_{1} w_{2} \in E(B(r))}\left[\operatorname{deg}\left(w_{1}\right) \times \operatorname{deg}\left(w_{2}\right)\right] \\
& =\prod_{w_{1} w_{2} \in E_{1}(B(r))}\left[\operatorname{deg}\left(w_{1}\right) \times \operatorname{deg}\left(w_{2}\right)\right] \\
& \times \quad \prod\left[\operatorname{deg}\left(w_{1}\right) \times \operatorname{deg}\left(w_{2}\right)\right] \\
& w_{1} w_{2} \in E_{2}(B(r)) \\
& =8^{\left|E_{1}(B(r))\right|} 16^{\left|E_{2}(B(r))\right|}=8^{\left(2^{r+2}\right)} 16^{\left(2^{r+2}(r-1)\right)}, \\
& M_{1}(B(r), x)=\sum_{w_{1} w_{2} \in E(B(r))} x^{\left[\operatorname{deg}\left(w_{1}\right)+\operatorname{deg}\left(w_{2}\right)\right]} \\
& =\sum_{w_{1} w_{2} \in E_{1}(B(r))} x^{\left[\operatorname{deg}\left(w_{1}\right)+\operatorname{deg}\left(w_{2}\right)\right]} \\
& +\sum_{w_{1} w_{2} \in E_{2}(B(r))} x^{\left[\operatorname{deg}\left(w_{1}\right)+\operatorname{deg}\left(w_{2}\right)\right]} \\
& =\sum_{w_{1} w_{2} \in E_{1}(B(r))} x^{6}+\sum_{w_{1} w_{2} \in E_{2}(B(r))} x^{8} \\
& =\left|E_{1}(B(r))\right| x^{6}+\left|E_{2}(B(r))\right| x^{8} \\
& =2^{r+2} x^{6}+2^{r+2}(r-1) x^{8} \text {, } \\
& \begin{aligned}
M_{2}(B(r), x) & =\sum_{w_{1} w_{2} \in E(B(r))} x^{\left[\operatorname{deg}\left(w_{1}\right) \times \operatorname{deg}\left(w_{2}\right)\right]} \\
& =\sum_{w_{1} w_{2} \in E_{1}(B(r))} x^{\left[\operatorname{deg}\left(w_{1}\right) \times \operatorname{deg}\left(w_{2}\right)\right]}
\end{aligned}
\end{aligned}
$$




$$
\begin{aligned}
& +\sum_{w_{1} w_{2} \in E_{2}(B(r))} x^{\left[\operatorname{deg}\left(w_{1}\right) \times \operatorname{deg}\left(w_{2}\right)\right]} \\
& =\sum_{w_{1} w_{2} \in E_{1}(B(r))} x^{8}+\sum_{w_{1} w_{2} \in E_{2}(B(r))} x^{16} \\
& =\left|E_{1}(B(r))\right| x^{8}+\left|E_{2}(B(r))\right| x^{16} \\
& =2^{r+2} x^{8}+2^{r+2}(r-1) x^{16} .
\end{aligned}
$$

\section{Fullerene networks}

The discovery of the fullerene molecules and related forms of carbon such as nanotubes has generated an explosion of activity in chemistry, physics, and materials science. A classical fullerene is an all-carbon molecule in which the atoms are arranged on a pseudospherical framework made up entirely of pentagons and hexagons, [27]. Its molecular graph is a finite trivalent graph embedded on the surface of a sphere with only hexagonal and (exactly 12) pentagonal faces. Deza et. al. [8] considered fullerenes' extension to other closed surfaces and showed that only four surfaces are possible, namely sphere, torus, Klein bottle and projective plane. Unlike spherical fullerenes, toroidal and Klein bottle's fullerenes have been regarded as tessellations of entire hexagons on their surfaces since they must contain no pentagons, see $[8,30]$.

\subsection{Toroidal and Klein-bottle fullerene networks}

Let $L$ be a regular hexagonal lattice and $P_{m}^{n}$ be an $m \times n$ quadrilateral section (with $m \geq 2$ hexagons on the top and bottom sides and $n \geq 2$ hexagons on the lateral sides with $n$ is even) cuts from the regular hexagonal lattice $L$, see Fig. 3. First, identify two lateral sides of $P_{m}^{n}$ to form a cylinder. If we identify the top and bottom sides of $P_{m}^{n}$ such that we identify the vertices $u_{i}^{0}$ and $u_{i}^{n}$, and the vertices $v_{i}^{0}$ and $v_{i}^{n}$ for $i=1,2, \cdots, m$, we are able to obtain the toroidal fullerene (toroidal polyhex) $\mathbb{H}_{m}^{n}$ with $m n$ hexagons, [3]. We can see that the toroidal fullerene is a cubic bipartite graph embedded on the torus such that each face is a hexagon. If we identify the top and bottom sides of $P_{m}^{n}$ in such a way that we identify the vertices $u_{1}^{0}$ and $u_{1}^{n}$, the vertices $u_{i}^{0}$ and $u_{m+2-i}^{n}$ for $i=2,3, \cdots, m$, and the vertices $v_{i}^{0}$ and $v_{m+1-i}^{n}$ for $i=1,2, \cdots, m$, we obtain the Klein-bottle fullerene (Klein-bottle polyhex) $\mathbb{K B}_{m}^{n}$ with $m n$ hexagons. In this case $\mathbb{K B}_{m}^{n}$ is a cubic bipartite graph of order $2 m n$ and size $3 m n$ embedded 
on the Klein-bottle and contains only hexagons.

Now we compute hyper-Zagreb index $H M(G)$, first multiple Zagreb index $P M_{1}(G)$, second Multiple Zagreb index $P M_{2}(G)$ together with Zagreb polynomials $M_{1}(G, x)$ and $M_{2}(G, x)$ for toroidal fullerene $\mathbb{H}_{m}^{n}$ and Klein-bottle fullerene $\mathbb{K B}_{m}^{n}$.

Theorem 3. Let $\mathbb{H}_{m}^{n}$ be the toroidal fullerene and $\mathbb{K B}_{m}^{n}$ be the Kleinbottle fullerene, for $n \geq 2$ even and $m \geq 2$. Then

$$
\begin{aligned}
H M\left(\mathbb{H}_{m}^{n}\right)=H M\left(\mathbb{K B}_{m}^{n}\right) & =108 m n \\
P M_{1}\left(\mathbb{H}_{m}^{n}\right)=P M_{1}\left(\mathbb{K B}_{m}^{n}\right) & =6^{3 m n} \\
P M_{2}\left(\mathbb{H}_{m}^{n}\right)=P M_{2}\left(\mathbb{K B}_{m}^{n}\right) & =9^{3 m n} \\
M_{1}\left(\mathbb{H}_{m}^{n}, x\right)=M\left(\mathbb{K B}_{m}^{n}, x\right) & =3 m n x^{6} \\
M_{2}\left(\mathbb{H}_{m}^{n}, x\right)=M_{2}\left(\mathbb{K B}_{m}^{n}, x\right) & =3 m n x^{9} .
\end{aligned}
$$

Proof. The toroidal fullerene $\mathbb{H}_{m}^{n}$ and also the Klein-bottle fullerene $\mathbb{K B}_{m}^{n}$ are of size $3 m n$, i.e. $\left|E\left(\mathbb{H}_{m}^{n}\right)\right|=\left|E\left(\mathbb{K B}_{m}^{n}\right)\right|=3 m n$. The considered fullerenes (polyhexes) are cubic graphs, thus $\operatorname{deg}\left(w_{1}\right)=3$ for every vertex $w_{1}$, i.e. the set of all neighbours is the same for every vertex. Using equations (1)-(5), we conclude that

$$
\begin{aligned}
H M\left(\mathbb{H}_{m}^{n}\right)=H M\left(\mathbb{K B}_{m}^{n}\right) & =\sum_{w_{1} w_{2} \in E\left(\mathbb{H}_{m}^{n}\right)}\left[\operatorname{deg}\left(w_{1}\right)+\operatorname{deg}\left(w_{2}\right)\right]^{2} \\
& =6^{2}\left|E\left(\mathbb{H}_{m}^{n}\right)\right|=108 m n, \\
P M_{1}\left(\mathbb{H}_{m}^{n}\right)=P M_{1}\left(\mathbb{K B}_{m}^{n}\right) & =\prod_{w_{1} w_{2} \in E\left(\mathbb{H}_{m}^{n}\right)}\left[\operatorname{deg}\left(w_{1}\right)+\operatorname{deg}\left(w_{2}\right)\right] \\
& =6^{\left|E\left(\mathbb{H}_{m}^{n}\right)\right|}=6^{3 m n}, \\
P M_{2}\left(\mathbb{H}_{m}^{n}\right)=P M_{2}\left(\mathbb{K B}_{m}^{n}\right) & =\prod_{w_{1} w_{2} \in E\left(\mathbb{H}_{m}^{n}\right)}\left[\operatorname{deg}\left(w_{1}\right) \times \operatorname{deg}\left(w_{2}\right)\right] \\
& =9^{\left|E\left(\mathbb{H}_{m}^{n}\right)\right|}=9^{3 m n},
\end{aligned}
$$

and hence the corresponding polynomials are

$$
M_{1}\left(\mathbb{H}_{m}^{n}, x\right)=M_{1}\left(\mathbb{K}_{m}^{n}, x\right)=\sum_{w_{1} w_{2} \in E\left(\mathbb{H}_{m}^{n}\right)} x^{\left[\operatorname{deg}\left(w_{1}\right)+\operatorname{deg}\left(w_{2}\right)\right]}
$$



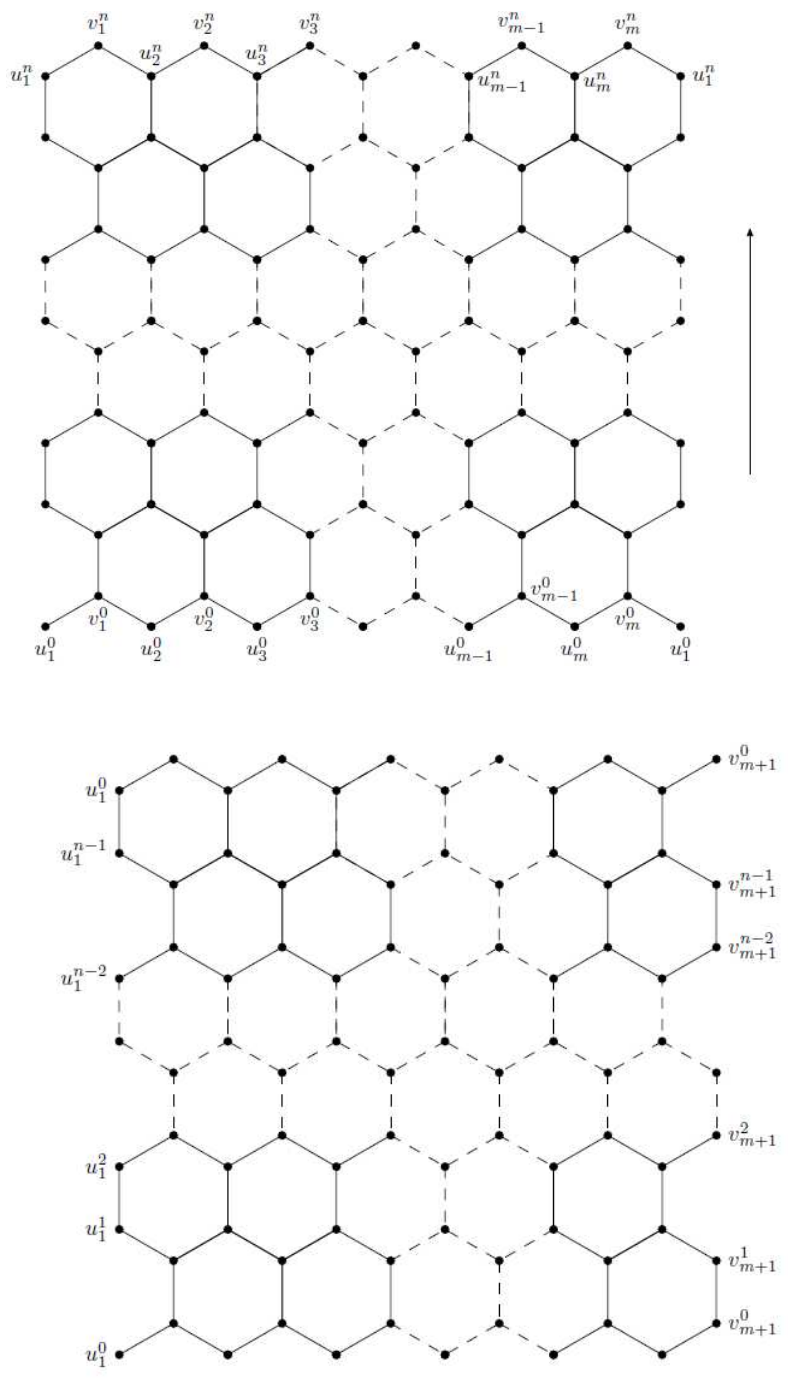

Figure 3: Quadrilateral sections $P_{m}^{n}$ (left) and $P_{(m+1) / 2}^{n}$ (right) cuts from the regular hexagonal lattice.

$$
=\sum_{w_{1} w_{2} \in E\left(\mathbb{H}_{m}^{n}\right)} x^{6}=3 m n x^{6}
$$


and

$$
\begin{aligned}
M_{2}\left(\mathbb{H}_{m}^{n}, x\right)=M_{2}\left(\mathbb{K B}_{m}^{n}, x\right) & =\sum_{w_{1} w_{2} \in E\left(\mathbb{H}_{m}^{n}\right)} x^{\left[\operatorname{deg}\left(w_{1}\right) \times \operatorname{deg}\left(w_{2}\right)\right]} \\
& =\sum_{w_{1} w_{2} \in E\left(\mathbb{H}_{m}^{n}\right)} x^{9}=3 m n x^{9}
\end{aligned}
$$

\subsection{Klein-bottle polyhex networks}

Now, let $L$ be a regular hexagonal lattice and $P_{(m+1) / 2}^{n}$ be a quadrilateral section (with $(m+1) / 2$ hexagons on the top and bottom sides, $m \geq 1$, and $n \geq 2$ hexagons on the lateral sides with $n$ is even) cut from the regular hexagonal lattice $L$, see Fig. 3. First, identify two lateral sides of $P_{m}^{n}$ to form a cylinder. If we identify the top and bottom sides of $P_{m}^{n}$ such that we identify the vertices $u_{i}^{0}$ and $u_{i}^{n}$, and the vertices $v_{i}^{0}$ and $v_{i}^{n}$ for $i=1,2, \cdots, m$, we are able to obtain the toroidal fullerene (toroidal polyhex) $\mathbb{H}_{m}^{n}$ with $m n$ hexagons, [3]. We can see that the toroidal fullerene is a cubic bipartite graph embedded on the torus such that each face is a hexagon. We identify the top and bottom sides of $P_{(m+1) / 2}^{n}$ to form a cylinder. Then we identify the lateral sides of cylinder such that we identify the vertices $u_{1}^{0}$ and $v_{m+1}^{0}$, and the vertices $u_{1}^{j}$ and $v_{m+1}^{n-j}$, for $j=1,2, \cdots, n-1$, to obtain the Klein-bottle polyhex $\mathbb{K B}_{(m+1) / 2}^{n}$. We can see that $\mathbb{K B}_{(m+1) / 2}^{n}$ is a cubic non-bipartite graph of order $2 n((m+1) / 2)$ and size $\frac{6 m n+3 n}{2}$ embedded on the Klein-bottle and contains $n((m+1) / 2)$ hexagons.

Now we compute hyper-Zagreb index $H M(G)$, first multiple Zagreb index $P M_{1}(G)$, second multiple Zagreb index $P M_{2}(G)$, Zagreb polynomials $M_{1}(G, x)$ and $M_{2}(G, x)$ for Klein-bottle polyhex $\mathbb{K B}_{(m+1) / 2}^{n}$.

Theorem 4. Let $\mathbb{K B}_{(m+1) / 2}^{n}$ be the Klein-bottle polyhex network for $n \geq 2$ even and $m \geq 2$. Then

$$
\begin{aligned}
H M\left(\mathbb{K B}_{m+1) / 2}^{n}\right) & =108 m n+54 n \\
P M_{1}\left(\mathbb{K B}_{(m+1) / 2}^{n}\right) & =6^{\frac{3 m n+3 n}{2}} \\
P M_{2}\left(\mathbb{K B}_{(m+1) / 2}^{n}\right) & =9^{\frac{3 m n+3 n}{2}} \\
M_{1}\left(\mathbb{K B}_{(m+1) / 2}^{n}, x\right) & =\left(\frac{3 m n+3 n}{2}\right) x^{6} \\
M_{2}\left(\mathbb{K B}_{(m+1) / 2}^{n}, x\right) & =\left(\frac{3 m n+3 n}{2}\right) x^{9} .
\end{aligned}
$$


Proof. The Klein-bottle polyhex $\mathbb{K}_{(m+1) / 2}^{n}$ contains $\frac{6 m n+3 n}{2}$ edges. The considered fullerenes (polyhexes) are cubic graphs, thus $\operatorname{deg}\left(w_{1}\right)=3$ for every vertex $w_{1}$, i.e. the set of all neighbors is the same for every vertex. Using equations (1)-(5), we have

$$
\begin{aligned}
& H M\left(\mathbb{K B}_{(m+1) / 2}^{n}\right)=\sum_{w_{1} w_{2} \in E\left(\mathbb{K B}_{(m+1) / 2}^{n}\right)}\left[\operatorname{deg}\left(w_{1}\right)+\operatorname{deg}\left(w_{2}\right)\right]^{2} \\
& =6^{2}\left|E\left(\mathbb{K B}_{(m+1) / 2}^{n}\right)\right|=108 m n+54 n, \\
& P M_{1}\left(\mathbb{K B}_{(m+1) / 2}^{n}\right)=\prod_{w_{1} w_{2} \in E\left(\mathbb{K B}_{(m+1) / 2}^{n}\right)}\left[\operatorname{deg}\left(w_{1}\right)+\operatorname{deg}\left(w_{2}\right)\right] \\
& =6^{\left|E\left(\mathbb{K B}_{(m+1) / 2}^{n}\right)\right|}=6^{\frac{6 m n+3 n}{2}}, \\
& P M_{2}\left(\mathbb{K B}_{(m+1) / 2}^{n}\right)=\prod_{w_{1} w_{2} \in E\left(\mathbb{K B}_{(m+1) / 2}^{n}\right)}\left[\operatorname{deg}\left(w_{1}\right) \times \operatorname{deg}\left(w_{2}\right)\right] \\
& =9^{\left|E\left(\mathbb{K B}_{(m+1) / 2}^{n}\right)\right|}=9^{\frac{6 m n+3 n}{2}} \text {, } \\
& M_{1}\left(\mathbb{K B}_{(m+1) / 2}^{n}, x\right)=\sum_{w_{1} w_{2} \in E\left(\mathbb{K B}_{(m+1) / 2}^{n}\right)} x^{\left[\operatorname{deg}\left(w_{1}\right)+\operatorname{deg}\left(w_{2}\right)\right]} \\
& =\sum_{w_{1} w_{2} \in E\left(\mathbb{K B}_{(m+1) / 2}^{n}\right)} x^{6}=\frac{6 m n+3 n}{2} x^{6}, \\
& \begin{aligned}
M_{2}\left(\mathbb{K B}_{(m+1) / 2}^{n}, x\right) & =\sum_{w_{1} w_{2} \in E\left(\mathbb{K B}_{(m+1) / 2}^{n}\right)} x^{\left[\operatorname{deg}\left(w_{1}\right) \times \operatorname{deg}\left(w_{2}\right)\right]} \\
& =\sum_{w_{1} w_{2} \in E\left(\mathbb{K B}_{(m+1) / 2}^{n}\right)} x^{9}=\frac{6 m n+3 n}{2} x^{9} .
\end{aligned}
\end{aligned}
$$




\section{Acknowledgement}

This work was supported by the Teaching Groups in Anhui Province (2016jytd080); the Natural Science Foundation of the Education Department of Anhui Province (KJ2019A1303); the Key Program of the Excellent Young Talents Support of Higher Education in Anhui Province (gxyq2018116); the Natural Science Foundation of Bozhou University (BYZ2018B03).

\section{References}

[1] T. Al-Fozan, P. Manuel, I. Rajasingh, R. S. Rajan, A new technique to compute Padmakar-Ivan index and Szeged index of pericondensed benzenoid graphs, J. Comput. Theor. Nanosci. 11 (2014), 533-539.

[2] A. Alwardi, A. Alqesmah, R. Rangarajan, I. N. Cangul, Entire Zagreb indices of graphs, Discrete Mathematics, Algorithms and Applications, 10, No 3 (2018), Art. 1850037 (16 pages); DOI: 10.1142/S1793830918500374.

[3] M. Bača, A. Shabbir, Total labeling of toroidal polyhexes, Sci. Int.(Lahore) 24, No 3 (2012), 239-241.

[4] V. E. Beneš, Mathematical Theory of Connecting Networks and Telephone Traffic, Academic Press (1965).

[5] A. R. Bindusree, I. N. Cangul, V. Lokesha, A. S. Cevik, Zagreb polynomials of three graph operators, Filomat 30, No 7 (2016), 1979-1986.

[6] K. C. Das, N. Akgunes, M. Togan, A. Yurttas, I. N. Cangul, A. S. Cevik, On the first Zagreb index and multiplicative Zagreb coindices of graphs, Analele Stiint. ale Universitatii Ovidius Constanta, 24, No 1 (2016), 153176.

[7] K. C. Das, A. Yurttas, M. Togan, I. N. Cangul, A. S. Cevik, The multiplicative Zagreb indices of graph operations, J. of Inequalities and Applications, 90 (2013), 1-14; doi:10.1186/1029-242X-2013-90.

[8] M. Deza, P. W. Fowler, A. Rassat, K. M. Rogers, Fullerenes as tilings of surfaces, J. Chem. Inf. Comput. Sci., 40 (2000) 550-558.

[9] A. A. Dobrynin, R. Entringer, I. Gutman, Wiener index of trees: Theory and applications, Acta Appl. Math., 66 (2001) 211-249. 
[10] M. Eliasi, A simple approach to order the multiplicative Zagreb indices of connected graphs, Trans. Comb., 4 (2012), 17-24.

[11] M. Eliasi, A. Ghalavand, Ordering of trees by multiplicative second Zagreb index, Trans. Comb., 5, No 1 (2016), 49-55.

[12] M. Eliasi, A. Iranmanesh, I. Gutman, Multiplicative version of first Zagreb index, MATCH Commun. Math. Comput. Chem., 68 (2012), 217-230.

[13] E. Estrada, L. Torres, L. Rodriguez, I. Gutman, An atom-bond connectivity index: modeling the enthalpy of formation of alkanes, Indian J. Chem., 37 (1998), 849-855.

[14] F. Falahati-Nezhad, A. Iranmanesh, A. Tehranian, M. Azari, Comparing the second multiplicative Zagreb coindex with some graph invariants, Trans. Comb., 3, No 4 (2014), 31-41.

[15] B. Furtula, I. Gutman, M. Dehmer, On structure-sensitivity of degreebased topological indices, Appl. Math. Comput., 219 (2013), 8973-8978.

[16] M. Ghorbani, N. Azimi, Note on multiple Zagreb indices, Iran. J. Math. Chem., 3, No 2 (2012), 137-143.

[17] A. Graovac, M. Ghorbani, M. A. Hosseinzadeh, Computing fifth geometric arithmetic index for nanostar dendrimers, J. Math. Nanosci., 1 (2011), $33-42$.

[18] I. Gutman, Degree-based topological indices, Croat. Chem. Acta, 86 (2013), 351-361.

[19] I. Gutman, K. C. Das, Some properties of the second Zagreb index, MATCH Commun. Math. Comput. Chem., 50 (2004), 103-112.

[20] I. Gutman, M. Kamran Jamil, N. Akhter, Graphs with fixed number of pendent vertices and minimal first Zagreb index, Trans. Comb., 4, No 1 (2015), 43-48.

[21] I. Gutman, O. E. Polansky, Mathematical Concepts in Organic Chemistry, Springer-Verlag, New York (1986).

[22] I. Gutman, N. Trinajstic, Graph theory and molecular orbitals. Total $\phi$ electron energy of alternant hydrocarbons, Chem. Phys. Lett., 17 (1972), $535-538$. 
[23] N. Habibi, T. Dehghan-Zadeh, A. R. Ashrafi, Extremal tetracyclic graphs with respect to the first and second Zagreb indices, Trans. Comb., 5, No 4 (2016), 35-55.

[24] S. Hayat, M. Imran, Computation of certain topological indices of nanotubes covered by $C_{5}$ and $C_{7}, J$. Comput. Theor. Nanosci. 12 (2015), $1-9$.

[25] S. Hayat, M. Imran, Computation of topological indices of certain networks, Appl. Math. Comput., 240 (2014), 213-228.

[26] S. A. Hosseini, M. B. Ahmadi, I. Gutman, Kragujevac trees with minimal atom-bond connectivity index, MATCH Commun. Math. Comput. Chem., 71 (2014), 5-20.

[27] S. Imran, M. Hussain, M. K. Siddiqui, M. Numan, Super face d-antimagic labeling for disjoint union of toroidal fullerenes, . Math. Chem., 55, No 3 (2017), 849-863.

[28] R. Kazemi, Probabilistic analysis of the first Zagreb index, Trans. Comb., 2, No 2 (2013), 35-40.

[29] M. Imran, S. Hayat, M. Y. H. Mailk, On topological indices of certain interconnection networks, Appl. Math. Comput. 244 (2014), 936-951.

[30] D. J. Klein, Elemental benzenoids, J. Chem. Inf. Comput. Sci., 34 (1994), 453-459.

[31] S. Konstantinidou, The selective extra stage butterfly, IEEE Trans. Very Large Scale Integr. (VLSI) Syst., 1 (1992), 502-506.

[32] X. Liu, Q. P. Gu, Multicasts on WDM all-optical butterfly networks, J. Inf. Sci. Eng., 18 (2002), 1049-1058.

[33] P. D. Manuel, M.I. Abd-El-Barr, I. Rajasingh, B. Rajan, An efficient representation of Benes networks and its applications, J. Discrete Algorithms, 6 (2008), 11-19.

[34] J. Rada, R. Cruz, I. Gutman, Benzenoid systems with extremal vertexdegree-based topological indices, MATCH Commun. Math. Comput. Chem., 72 (2014), 125-136. 
[35] P. S. Ranjini, V. Lokesha, I. N. Cangul, On the Zagreb Indices of the Line Graphs of the Subdivision Graphs, Appl. Math. and Comput., 218, No 3 (2011), 699-702.

[36] G. H. Shirdel, H. RezaPour, A. M. Sayadi, The hyper-Zagreb Index of graph operations, Iran. J. Math. Chem. 4, No 2 (2013), 213-220.

[37] M. K. Siddiqui, M. Imran, A. Ali, On Zagreb indices, Zagreb polynomials of some nanostar dendrimers, Appl. Math. Comput., 280 (2016), 132-139.

[38] M. Togan, A. Yurttas, I. N. Cangul, All versions of Zagreb indices and coindices of subdivision graphs of certain graph types, Advanced Studies in Contemp. Math., 26, No 1 (2016), 227-236.

[39] M. Togan, A. Yurttas, I. N. Cangul, Zagreb and multiplicative Zagreb indices of $r$-subdivision graphs of double graphs, Scientia Magna, 12, No 1 (2017), 115-119.

[40] H. Wiener, Structural determination of paraffin boiling points, J. Amer. Chem. Soc., 69 (1947), 17-20.

[41] A. William, A. Shanthakumari, Minimum cycle covers of butterfly and Benes network, Int. J. Math. Soft Comput., 2, No 1 (2012), 93-98.

[42] J. Xu, Topological Structure and Analysis of Interconnection Networks, Kluwer Academic Publishers (2001). 